\title{
Paleoecology, Evolutionary Approaches to
}

\author{
L. C. BISHOP \\ Liverpool John Moores University, United Kingdom
}

Paleoecology is the study of the relationships between organisms and their environments in the past. The principal application of paleoecology in anthropology is to the study of human evolution. Paleoecology provides the context to our study of changes in biological and behavioral adaptation through evolutionary time, such that the major developments which define our differences from our nearest relatives can be characterized in context and the development of human distinctiveness can be examined within an environmental framework. The term derives from the ancient Greek $\pi \alpha \lambda \alpha i o ́ s$ (palaiós, "old") and the German Ökologie, from ancient Greek oĩ

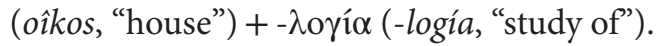

All organisms interact with their environments and ecology is the study of those interactions. Environments are both physical and biological and characterizing the dynamic relationships between factors is one of the main challenges of studying ecology in the present as well as in the past. The physical environment includes such variables as temperature, rainfall, and aspects of the physical setting, such as altitude, soil characteristics, and local geography, including availability and characteristics of fresh water locally. The biotic environment consists of all the other living things, both plant and animal, with which organisms interact. Paleoecology deals with these things in the past, which makes them even harder to study than in the present since we are more limited in how we can investigate these relationships. Those seeking to characterize ecology in the past often rely on proxy measures of both biotic and abiotic factors in an imperfect geological record.

Paleoecology helps us to understand the context of human evolution, particularly as it can relate to overall ecosystem changes that provide the background for adaptation. The major developments in human evolution which distinguish us from our closest relatives, such as general reduction in dentition, the development of bipedal locomotion, and increasing brain size, can all be viewed in an ecological context as responses to environmental constraints which favored new morphologies and behaviors. Throughout the history of paleoanthropology, various hypotheses have been used as investigative frameworks for studies of ecological relationships, from the "savanna hypothesis" derived from the initial writings of Charles Darwin (1871), through Elizabeth Vrba's "turnover-pulse hypothesis" (1985) linking large-scale global climate change to pulses in extinction and speciation driving changes in hominin faunal communities. A dominant ecological hypothesis today is Richard Potts's "variability selection hypothesis" (1998), which predicts that behavioral variability and novelty is favored in highly variable environmental settings. 
The study of ecology—and, by extension, paleoecology-has two major branches. The first is autecology (ancient Greek via Latin auto- ["on itself"] + ecology) and this is the study of the interactions between one type of organism (e.g., a species) and its environment, for example, we can study the interactions between an extinct hominin species, Homo erectus, and its environment, examining its relationships with predators, prey, and other animals and plants within a reconstruction of its abiotic environment. The second is synecology (from ancient Greek $\sigma v v$ - [sun-], from $\sigma \delta v$ [sún, "with, in company with, together with"]) and this focuses on the study of groups of organisms associated as a unit. Usually the groups are along the lines of a defined portion of the biological community (e.g., large mammals or carnivores) which can be examined as a more or less coherent unit. For example, since all carnivores are dependent on animal prey to a greater or lesser extent, they can be considered as a group which together shares important ecological characteristics, which in turn means they would have similar interactions with their biotic and abiotic environment.

Many studies of paleoecology rely on the principle of uniformitarianism, a term borrowed from geology which means that the physical processes of the past are the same as those observable in the present, often summarized with the observation that the present is the key to the past. This approach is helpful in that it gives confidence that analogy between past and present ecosystems has a well-founded basis. However, we can easily observe via our knowledge of past faunas and floras that change in many respects has occurred. Since the physical laws are the same, some or more aspects of conditions must have changed to create these differences. The most universal driver is climatic change on a global scale, although continental and regional changes can also cause paleoecological change visible in the make-up of the ecosystem's constituent biological elements.

Paleoecological investigations usually focus on a particular geographical or temporal range associated with the limits and scale of the ecosystem under investigation. An ecosystem includes all interconnected biotic and abiotic components in a geographic area, the size of which is usually constrained by both the nature of the research question and the availability of evidence. The geological record is both patchy through space and incomplete through time and its limits provide the primary constraint on the questions that can be answered. Another major constraint on the study of paleoecology is that of taphonomy, the processes that occur and biases which affect how organisms are preserved in the fossil record and how the resulting fossils are recovered and become available for study. Taphonomic processes bias the fossil record in various ways that alter how representative a fossil assemblage is of the living community it preserves. Therefore, a good understanding of the taphonomy of fossil assemblages is a necessity when investigating paleoecology.

Another major challenge is that of chronology. When studying fossils derived from a single geological sequence in one region, creating a temporal framework may (or may not) be relatively straightforward. However, it can be difficult to correlate temporally different geological sequences distributed over larger areas, some of which may not be amenable to absolute or radiometric dating. Therefore examining large-scale paleoecological change, for example on a continental scale encompassing the entire known range of a species, can be a challenge, while examining a smaller geographical scale will 
give a limited, yet more easily definable, view of the ecological range of a species or community.

As befits a discipline as all-encompassing as paleoecology, there are many methods used to examine the relationships between organisms and their biotic and abiotic environment. Many of these take the approach of characterizing different aspects of these relationships and interactions: for example, feeding ecology. To examine the feeding ecology of a herbivorous mammalian species, one might look at the morphology of its teeth to determine the type of diet it likely had. A less general way of looking at diet would focus on changes made to an animal's dentition by its actual diet, either macroscopically (using a method called mesowear analysis, which examines how teeth are changed attritionally through wear) or microscopically (using microwear analysis, which examines the microscopic damage left on the surface of teeth by the foods eaten). Diet can also be examined through chemical analysis, particularly by analyzing the variation in the proportions of isotopes which indicate particular plant food diets (stable isotopes of carbon linked to plant photosynthetic pathways). To characterize the diet of a species, one might use several methods of analysis on numerous different specimens, either from one defined region or from a wider area, at one time or from several points in time, depending on the question addressed.

For students of paleoanthropology, another important aspect of paleoecology is the relationship of hominin species and other mammals to the vegetational environment. This may affect such diverse aspects of ecology as hominin diet and also predation pressure, since vegetation affects both plant and animal species available to eat and also the types of predators likely to be present and which prey they would favor. Overall vegetation structure can be studied by examining the proportions of stable carbon isotopes in the fossil soils, which, as in the case of the dietary studies, indicates the overall percentage of plants that used different photosynthetic pathways and created soil carbonates. Alternatively, vegetation can be inferred through fossils of plant leaves and seeds, which are rare but identifiable to taxon, or by the analysis of microfossils of plant pollen or phytoliths preserved in the soil. These relative proportions of both pollen and phytoliths are biased by the amount produced by each plant, which varies widely, and, in the case of pollen, how far it is dispersed. However, with these in mind, a profile of vegetation types can be reconstructed. An alternative synecological approach uses the entire faunal community to predict what vegetation structure it might have been supported by, usually by analogy to faunal communities living in modern wildlife preserves or protected areas.

It can be challenging to understand abiotic aspects of terrestrial environments, such as temperature, seasonality, and even aspects of past geography-altitude or the presence of fresh water. Of these, the last is usually most straightforward because water action plays a large role in the concentration, burial, and preservation of mammalian fossils. The nature of local water sources can be greatly illuminated by the study of sedimentology and, for lakes in particular, the micropaleontology of diatoms preserved in lacustrine sediments can help characterize lake salinity and $\mathrm{pH}$. Determination of local temperature is more complicated. Analysis of plant leaf morphology can also help determine local temperature and rainfall but plant leaves are very rarely preserved in the fossil record. Alternatively, when the age of the fossil assemblage is well constrained, temperature is often determined with reference to the long-term records of climate 
change preserved in ice cores or in marine records like deep-sea sediment cores, which show changes in the oxygen isotopes of foraminifera (marine microorganisms) and fluctuations in the quantities of African terrestrial dust related to temperature. However, temperature estimates must be taken with a pinch of salt because the connection between large-scale, often distant, indicators of global climate change and what is happening in any one terrestrial locale relies on inference alone.

Developments in paleoecology have three main drivers in discovery, methodology, and theory. Discovery of new fossils, species, or fossil deposits are only some of the factors which can lead to new revelations about paleoecology. New methods, often derived from other disciplines, can lead to new insights when they are applied to paleoanthropological contexts. Finally, new theoretical insights and hypotheses can change the focus of current research in paleoecology by providing new frameworks and points of reference for analysis and discussion.

SEE ALSO: Landscape; Homo, Evolution of the Genus; Environmental Anthropology; Climate Change; Hominins, Early; Social-Ecological Systems; Anthropology, Biological and Evolutionary; Human Paleoecology; Dating, Archaeological; Archaeological Approaches in Anthropology

\section{REFERENCES AND FURTHER READING}

Bobe, René, Zeresenay Alemseged, and Anna K. Behrensmeyer. 2007. Hominin Environments in the East African Pliocene: An Assessment of the Faunal Evidence. Vertebrate Paleobiology and Paleoanthropology Series, Vol. 1. Dordrecht: Springer.

Darwin, Charles. 1871. The Descent of Man and Selection in Relation to Sex. London: John Murray. Kingston, John D. 2007. "Shifting Adaptive Landscapes: Progress and Challenges in Reconstructing Early Hominid Environments.” Yearbook of Physical Anthropology 50: 20-58.

Potts, Richard. 1998. "Variability Selection in Hominid Evolution." Evolutionary Anthropology 7: 81-96.

Vrba, Elizabeth S. 1985. "Environment and Evolution: Alternative Causes of the Temporal Distribution of Evolutionary Events." South African Journal of Science 81: 229-36. 
Please note that the abstract and keywords will not be included in the printed book, but are required for the online presentation of this book which will be published on Wiley's own online publishing platform.

If the abstract and keywords are not present below, please take this opportunity to add them now.

The abstract should be a short paragraph of between 50 and 150 words in length and there should be at least 3 keywords.

\begin{abstract}
Paleoecology is the study of the relationships between organisms and their environments in the past. The principal application of paleoecology in anthropology is to the study of paleoanthropology. Paleoecology provides a framework for understanding human evolution within the context of changing environments, influenced and influencing both biotic and abiotic aspects of their surroundings.
\end{abstract}

\title{
KEYWORDS
}

climate change; ecology; environment; human evolution; landscape; paleoanthropology; physical/biological anthropology 